\title{
PREPARATION OF LANGMUIR-BLODGETT FILMS OF METHACRYLATE COPOLYMER BEARING AZO-DYE CHROMOPHORES AND THEIR NONLINEAR OPTICAL PROPERTIES
}

\author{
MASAO KATO ${ }^{1}$, TAKAYUKI OHONO ${ }^{1}$, CHIKARA ISHII ${ }^{1}$, \\ HIRO MATSUDA ${ }^{2}$ and HACHIRO NAKANISHI ${ }^{3}$ \\ ${ }^{1}$ Faculty of Industrial Science and Technology, Science University of Tokyo, \\ 2641 Yamazaki, Noda, Chiba 278, JAPAN, \\ 2 National Institute of Materials and Chemical Research, 1-1 Higashi, Tsukuba 305, JAPAN, \\ ${ }^{3}$ Institute for Chemical Reaction Science, Tohoku University, Sendai 980-77, JAPAN
}

\begin{abstract}
A methacrylate copolymer, poly(MO), was prepared from radical copolymerization of octadecyl methacrylate and methacrylamide bearing DR-1 type azo-dye chromophore as NLO active group, which was synthesized through 7 reaction steps. Poly(MO) gave LB film on quartz substrate by the vertical dipping method. Transfer of the monolayer at the water-air interface was easier in upstroke than in down stroke. By SHG measurement for the multilayers it was found that only in the first monolayer exists an effective noncentrosymmetric structure of NLO chromophores, showing SHG. Unexpectedly, when a heat treatment was performed SH intensity of the monolayer decreased, whereas that of the multilayers increased significantly. More detailed investigations about this heat response behavior were carried out.
\end{abstract}

\section{Introduction}

Langmuir Blodgett Film(LB) technique is considered one of the most promising method to fabricate noncentrosymmetric organic thin films, showing second harmonic generation(SHG). LB films of amphiphilic azo-dye have been investigated as the object of several nonlinear optical(NLO) studies[1-3]. Generally, structure of monomeric LB film does not exhibit higher mechanical and thermal stabilities, thereby degrading NLO properties. Meanwhile, this defect is expected to be improved if the noncentrosymmetric film is prepared by using an amphiphilic NLO polymer functionalized with azo-dye chromophores [4-7].

In this work, copolymer of octadecyl methacrylate(ODMA) and methacrylamide bearing DR-1 
type azo-dye chromophore(MAHNA) was synthesized as NLO polymer, and preparations and NLO properties of its LB films were investigated.

\section{Experimental}

\section{Synthesis}

4-[N-methyl-N-(6-methacrylaminohexyl)amino]-4'-nitroazobenzene(MAHNA), as NLO active monomer was synthesized as follows. 6-Iodo-1-hexanol (1) was synthesized by halogen exchange reaction between sodium iodide and 6-chloro-1-hexanol in acetone at $60^{\circ} \mathrm{C} . \quad 6-(\mathrm{N}-$ methylanilino)hexanol (2) was synthesized by the reaction of $\mathrm{N}$-methylaniline with 1 in dimethyl formamide at room temperature in the presence of sodium bicarbonate. [N-Methyl-N-(6phthalimidohexyl)] aniline (3) was synthesized by tosylation of 2 with tosylchloride, followed by, imination of the product with potassium phthalimide, according to the method described in the literature [8]. [(N-Methyl-N-(6-aminohexyl)]aniline (4) was synthesized by the reaction of 3 with hydrazine hydrate [9]. [(N-Methyl-N-(6-methacrylaminohexyl)]aniline (5) was synthesized by the reaction of 4 with methacrylic unhydride in dichloromethane-pyridine mixture at room temperature. MAHNA was synthesized by the reaction of 5 with diazotized p-nitroaniline (pH 3-4) at $0-5^{\circ} \mathrm{C}$. Recrystallization from methanol gave dark-red crystals, m.p. $141-142^{\circ} \mathrm{C}$.

${ }^{1} \mathrm{H}$ NMR $\left(\mathrm{CDCl}_{3}\right): \delta=8.31$ (triplet $\left.(\mathrm{s}), \mathrm{J}=4.6,2 \mathrm{H},-\mathrm{N}=\mathrm{N}-\mathrm{C}(\mathrm{CH})_{2}(\mathrm{CH})_{2} \mathrm{C}-\mathrm{NO}_{2}\right), 7.91$ (multiplet(m), $4 \mathrm{H},>\mathrm{N}-\mathrm{C}(\mathrm{CH})_{2}(\mathrm{CH})_{2} \mathrm{C}-\mathrm{N}=\mathrm{N}-\mathrm{C}(\mathrm{CH})_{2}(\mathrm{CH})_{2} \mathrm{C}-\mathrm{NO}_{2}$ ), 6.73 (doublet (d), J=9.6, 2H, $\left.>\mathrm{N}-\mathrm{C}(\underline{\mathrm{CH}})_{2}(\mathrm{CH})_{2} \mathrm{C}-\right), 5.80(\mathrm{~s}, 1 \mathrm{H},-\mathrm{CON} \underline{\mathrm{H}}-), 5.67,5.32\left(\mathrm{~s}, \mathrm{~s}, 2 \mathrm{H}, \underline{\mathrm{CH}}_{2}=\mathrm{C}\left(\mathrm{CH}_{3}\right) \mathrm{CO}-\right), 3.45(\mathrm{t}$, $\mathrm{J}=7.6,2 \mathrm{H},-\mathrm{CONH}-\mathrm{CH}_{2}$ ), 3.32 (quartet, J=6.8, 2H, $-\mathrm{CH}_{2}-\mathrm{N}$-phenyl), $3.09\left(\mathrm{~s}, 3 \mathrm{H}, \mathrm{CH}_{3}-\mathrm{N}-\right.$ phenyl), 1.96 (s, 3H, $\left.\mathrm{CH}_{2}=\mathrm{C}\left(\mathrm{CH}_{3}\right) \mathrm{CO}-\right)$, $1.39-1.65\left(\mathrm{~m}, 8 \mathrm{H}, \mathrm{CH}_{2}\left(\mathrm{CH}_{2}\right)_{4} \mathrm{CH}_{2}\right)$.

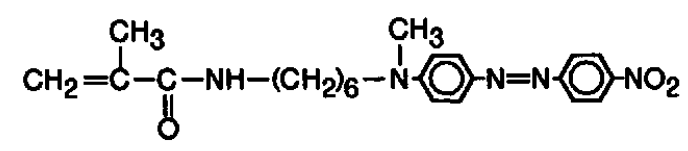

Octadecyl methacrylate (ODMA) was purchased from Tokyo Kasei and was purified by vacuum distillation just before use.

Copolymerization of MAHNA and ODMA (the mole ratio was 3:7) in tetrahydrofuran with the use of azobisisobutylonitrile as radical initiator was carried out in a vacuum sealed glass tube at $60^{\circ} \mathrm{C}$. The copolymer (poly (MO)) thus obtained was purified by reprecipitation from methanol (Yield 10.7\%). Copolymer composition calculated from the result of ${ }^{1} \mathrm{H}$ NMR measurement is 
shown in Fig. 1. Molecular weight of poly (MO) was $\bar{M} n=7100(\overline{\mathrm{M}} \mathbf{w} / \overline{\mathrm{M}} \mathrm{n}=1.61)$.

\section{Method and Measurements}

LB Film Preparation: A $0.5 \mathrm{wt} \%$ chloroform solution of poly(MO) was spread on water to form a thin layer. The layer was deposited onto quartz substrate by means of the LB vertical lifting method using a film balance controller (Science Keisoku FSD20).

\section{Characterization of Monomer and Copolymer:}

SHG of LB films was measured by the Maker-fringe method using a Q-switched Nd : YAG Laser (Spectra Physics DCR-2; $1064 \mathrm{~nm}, 8 \mathrm{~ns} /$ pules, $10 \mathrm{~mJ} /$ pulse). The second order coefficient, $d_{33}$, was evaluated by use of single-crystal quartz plate of $0.5 \mathrm{~mm}$ thickness $\left(d_{11}=8 \mathrm{x}\right.$

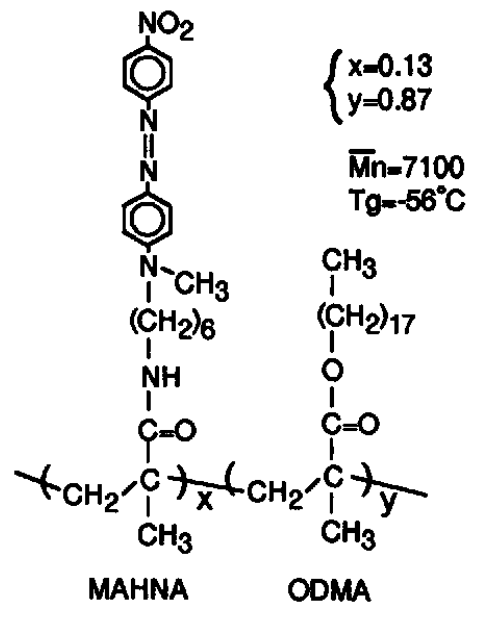

Fig.1 Structure of poly(MO) $\left.10^{-10} \mathrm{esu}\right)$. Refractive index was measured with a

Shimadzu AEP-100 ellipsometer. ${ }^{1} \mathrm{H}$ NMR spectra were recorded on a JEOL EX-400 spectrometer. UV-VIS spectra were obtained on a Shimadzu 3100-S UV-VIS spectrometer. Measurement of molecular weight was obtained using a Tosoh HLC-8020 gel-permeation chromatograph with tetrahydrofuran as eluent. Thermal analysis was performed using a Mettler DSC30 system with a heating rate of $20^{\circ} \mathrm{C} / \mathrm{min}$.

\section{Results and Discussion}

DSC chart of poly(MO) is shown in Fig. 2.A very weak endothermic peak at $-56^{\circ} \mathrm{C}$ due to $\mathrm{Tg}$ is observed.A sharp endothermic peak appears at $30^{\circ} \mathrm{C}$, which might be attributed to fusion of the long alkyl side chains.

The $\pi$-A curves of poly(MO) at different temperatures ranging from 10 to $25^{\circ} \mathrm{C}$ are illustrated in Fig. 3. Poly(MO) monolayer

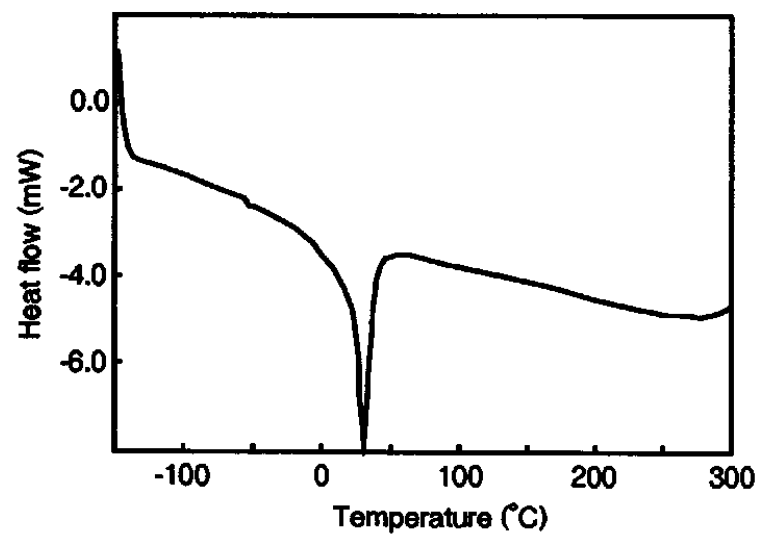

Fig. 2 DSC chart of poly(MO). spread on water at the temperature range could be deposited on substrate by the vertical dipping method maintaining the surface pressure at $20 \mathrm{mN} / \mathrm{m}$. The most preferable $\pi-A$ curve among them is considered to be one observed at $15^{\circ} \mathrm{C}$. 
From the curve the limiting area was calculated to be $32 \AA^{2} /$ unit.

Transfer of poly(MO) at waterair interface onto substrate was easier in upstroake than in downstroke. Namely, up-lift deposition ratio (= deposition during up-lift process /deposition during up-lift and down process) [10] of the film was 0.71 . As shown in Fig. 4, a linear relationship between the absorbance at $480 \mathrm{~nm}$ due to NLO chromophore

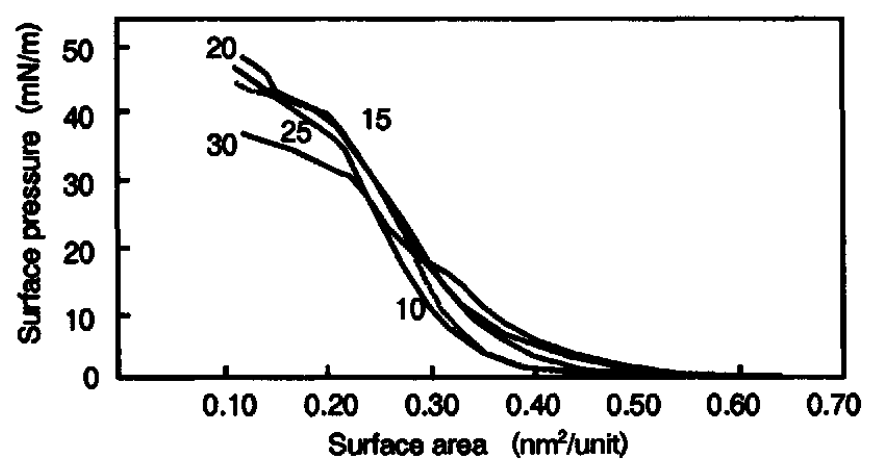

Fig.3 Surface pressure-area isotherms of poly $(\mathrm{MO})$ at $10,15,20,25$ and $30^{\circ} \mathrm{C}$.

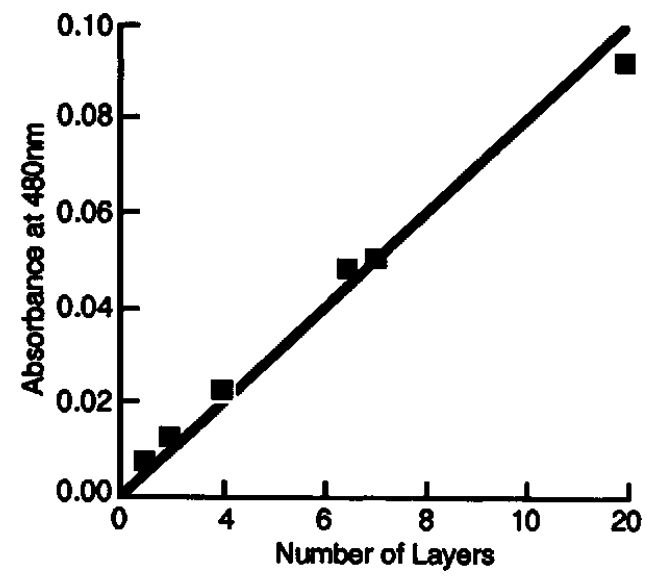

Fig.4 Relationship between absorbance at $480 \mathrm{~nm}$ and the number of layers.

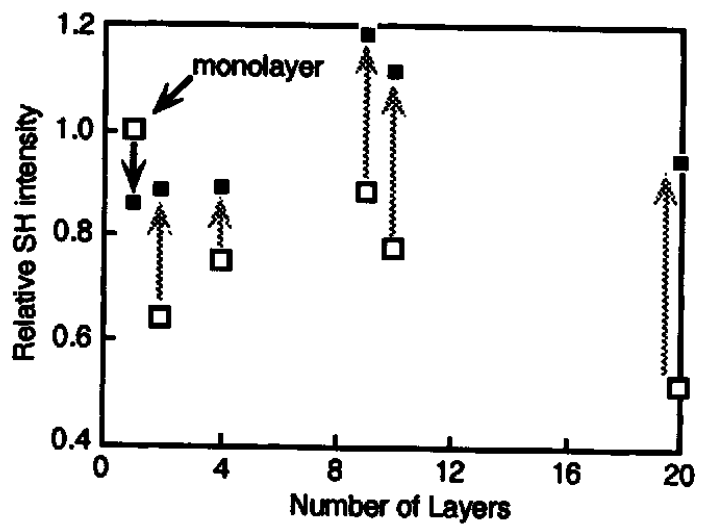

Fig.5 Heat responce of SH intensity related to the number of layers.

$\square$ : Before heat treatment

: After heat treatment at $60^{\circ} \mathrm{C}$ for $5 \mathrm{~min}$

multilayers (10 layers) reveals an interesting 
heat response behavior toward the repeated heating-cooling treatment. The SH intensity increased up to 3 times in contrast with original one when the heat treatment was performed at $50^{\circ} \mathrm{C}$ for $5 \mathrm{~min}$, followed by cooling at $22^{\circ} \mathrm{C}$. The phenomenon seems to occur by the fact that the orientation of NLO chromophores in the first monolayer is disordered by heating, however, after cooling it becomes much higher than before the heating as the result of the reorientationcrystallization of NLO chromophores.

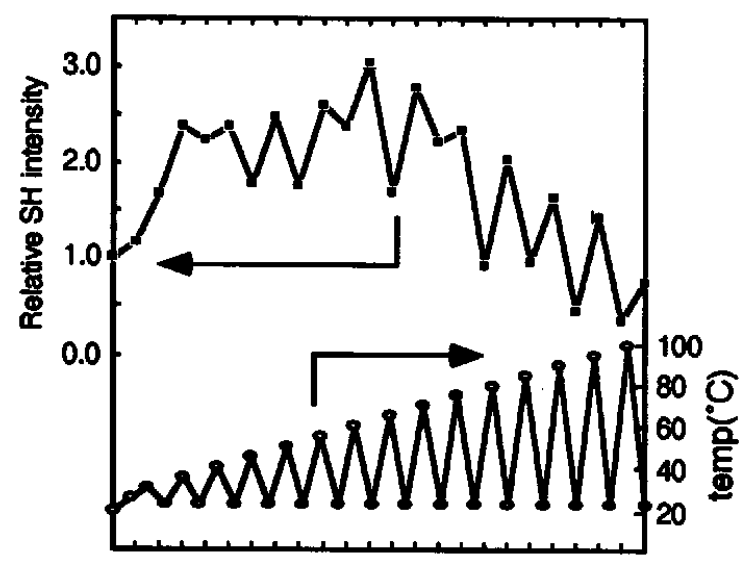

Fig.6 Changes in SH intensities of copolymer LB film(10 layers) by heat treatment. Heat treatment : Heating for $5 \mathrm{~min}$, and cooling $22^{\circ} \mathrm{C}$.

Probably, the upper layers exert a covering

effect to restrain thermal motion of the orientated NLO chromophores in the first monolayer at $22^{\circ} \mathrm{C}$ to give stable SHG. The heat treatment more than $50^{\circ} \mathrm{C} \mathrm{SH}$ intensity decreased with increasing temperature.

Average tilt angle of the orientated NLO chromophores can be given by the following calculation. Relationship between $d_{33}$ and average tilt angle, $\theta$, of NLO chromophores is expressed by eq. (1) [12].

$$
d_{33}=N \cdot f^{(2 \omega)} \cdot f^{(\omega)} \cdot f^{(\omega)} \cdot \beta x x x \cdot \cos ^{3} \theta
$$

where $f$ is the local field factor, $\beta \mathbf{x x x}$ is the second-order hyperpolazability and $\mathrm{N}$ is the NLO chromophore density. Since reflux index, $\mathrm{n}$, of the monolayer was measured to be $1.62(633 \mathrm{~nm})$, if $n \omega=n_{2 \omega}=n$ is assumed Lorentz correction factor defined as $f^{(\omega)}=\left(n_{\omega}^{2}+2\right) / 3$ will be expressed by $f^{(\omega)}=f^{(2 \omega)}=f=1.57$. Thus, eq. (1) is convertible to eq. (2).

$$
\cos ^{3} \theta=d_{33} / N \cdot f^{3} \cdot \beta x x x
$$

Here, $\beta x x x$ can be used that of DR-1 azo-dye $\left(1.2 \times 10^{-28} \mathrm{esu}[6]\right) . \mathrm{N}$ is $2.95 \times 10^{-20} \mathrm{~mol} \cdot \mathrm{cm}^{-3}$. By using eq. (2) average tilt angles of NLO chromophores depending on the number of poly(MO) monolayers before and after heat treatment at $60^{\circ} \mathrm{C}$ for $5 \mathrm{~min}$ were calculated and the result is demonstrated in Fig. 7. A reasonable correlation was obtained between Fig. 5 and Fig. 7.

\section{Conclusion}

The heat response behavior in SHG for poly(MO) multilayers is considered to occur by the fact 
the orientation of NLO chromophores in the first monolayers is disordered by heating, however, after cooling it becomes much higher than before the heating due to the reorientationcrystallization of NLO chromophores. Perhaps, the upper layers exert a covering effect to restrain thermal motion of the orientated NLO chromophores in the first monolayer at low temperature to give stable SHG.

\section{References}

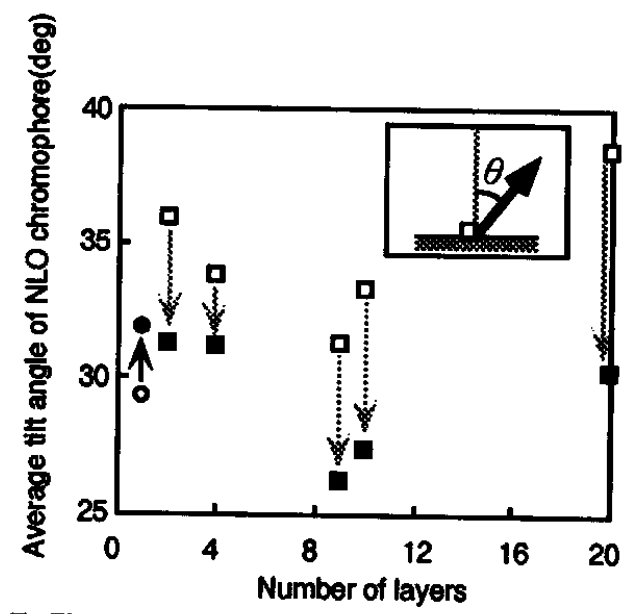

Fig.7 Changes in average tilt angles of NLO chromophores in poly(MO) LB film by heat treatment at $60^{\circ} \mathrm{C}$ for $5 \mathrm{~min}$.
0 : Monolayer, before heat treatment. $\mathrm{d} 33=92 \times 10^{-9} \mathrm{esu}$
- : Monolayer, after heat treatment. $\mathrm{d} 33=85 \times 10^{-9} \mathrm{esu}$
므: Multilayers, before heat treatment.
- : Multilayers, after heat treatment.

1. O.A.Aktsipetrov, N.N.Akhmediev, I.M.Baranova, E.D. Mishina and V.R.Novak, Sov. Tech.Lett. 11, 249(1993).

2. I.Ledoux, D.Josse, P.Viakovic, J.Zyss, P.A.Hahn, P.F.Gordon, B.D.Bothwell, S.K.Gupta, S.Allen, P.Robin, E.Chasting and J.C.Debois, Europhys.Lett. 3, 803(1987).

3. J. Loulergue, C.M.Dumont, Y.Levy, P.Robin, J.P.Pocholle and M.Popuchon, Thin Solid Films $160,399(1988)$.

4. N.Carr and M.J.Goodwin, Makromol.Chem.Rapid Commun. 8, 487(1987).

5. K.Kajikawa, T.Anzai, H.Takezoe, A.Fukuda, S.Okada, H.Matsuda, H.Nakanishi, T.Abe and H.Ito, Chem.Phys.Lett. 192, 113(1992).

6. K.Kajikawa, T.Yamaguchi, T.Anzai, H.Takezoe, A.Fukuda, S.Okada, H.Matsuda, T.Abe and H.Ito, Langmuir, 8, 2764(1992).

7. T.Verbiest, C.Samyn and A.Persoons, Thin Solid Films 242, 139(1994).

8. M.Mutter, Tetrahydron Lett. No.31, 2839(1978).

9. C.S.Marvel and V.C.Sekera, "Organic Syntheses, Coll. Vol.3", John Wiely \& Sons Inc. (1955) p.151.

10. Y.Yokoh and N.Ogata, PolymJ., 24, 63(1992).

11. G.Duda, A.J.Schouten, T.Arndt, G.Lieser, G.F.Schmid, C.Bubeck and G.Wegner, Thin Solid Films , 159, 221(1988).

12. D.S.Chemla, J.L.Oudar and J.Jerphagon, Phys.Rev, , 42, 4534(1975). 